\title{
Estructura de Capital, desde la perspectiva del Trade-Off, a nivel Latinoamérica (2008 - 2016).
}

\author{
Cervantes Monsreal Aníbal Humberto[a], Pérez Castañeda Suly Sendy[a], Cruz Ramírez Dorie ${ }^{[a], ~ S a u z a ~}$ \\ Ávila Beatriz[a]
}

[a]Profesores Investigadores de la Escuela Superior de Cd. Sahagún de la Universidad Autónoma del Estado de Hidalgo

\section{RESUMEN}

Este trabajo se dedica fundamentalmente al análisis comparativo de artículos científicos elaborados entre los años 2008 y 2016, que evalúan el comportamiento financiero bajo el enfoque de la teoría del Trade-off, en empresas de diferentes sectores, en países de Latinoamérica. Este análisis se orienta inicialmente a la investigación de tipo documental, para recabar información científica de estudios realizados, para poder generar un comparativo del objetivo esperado, con el resultado alcanzado en cada uno de los casos; luego se genera un análisis descriptivo considerando variables de análisis como el sector al que pertenecen las empresas analizadas, el país donde se desarrolló el estudio, el año dentro del parámetro determinado para analizar, la metodología aplicada y los resultados obtenidos dentro de cada artículo.

PALABRAS CLAVE: Trade-off, Latinoamérica, Finanzas.

\begin{abstract}
This work is mainly devoted to the comparative analysis of scientific articles produced between 2008 and 2016, which evaluate the financial behavior under the Trade-off theory approach, in companies of different sectors, in Latin American countries. This analysis is initially oriented to documentary research, to collect scientific information from studies carried out, in order to generate a comparison of the expected objective, with the result achieved in each case; Then a descriptive analysis is generated considering analysis variables such as the sector to which the companies analyzed belong, the country where the study was carried out, the year within the parameter determined to analyze, the methodology applied and the results obtained within each article.
\end{abstract}

KEY WORDS: Trade-off, Latin America, Finance. 


\section{INTRODUCCIÓN}

La investigación en el área financiera ha evolucionado desarrollándose alrededor de distintos temas de interés, de los cuales, nos enfocamos en la Teoría del Trade-Off, dentro de la Estructura de Capital, para lo cual se hizo un análisis comparativo de artículos científicos, publicados en países Latinoamericanos en un lapso del año 2008 al 2016, que aplicaron esta teoría en empresas de distintos sectores de mercado, con el fin de verificar si su comportamiento financiero, responde al objetivo de la teoría antes mencionada, el cual dice que, la combinación de fuentes de financiamiento permite minimizar el costo de capital, y maximizar el valor de mercado de la empresa (Tenjo, 2011).

Este estudio se realizó considerando variables de análisis, como el país donde se aplicó el estudio tomando en cuenta que pertenece a Latinoamérica, el año en que se desarrolló la investigación del artículo publicado, el cual se encuentra el lapso que se estableció en esta investigación de carácter comparativa, la metodología que se aplicó dentro de cada uno de los trabajos estudiados y los resultados obtenidos.

Finalmente, después de generar un análisis comparativo, se obtuvieron elementos de análisis interesantes, los cuales nos indican el comportamiento de las empresas bajo el enfoque del Trade-Off, además de poder identificar de manera precisa a qué tipo de financiamiento recurren, según el tamaño de organización.

\section{MARCO TEÓRICO}

El Trade-Off, es una teoría propuesta por Stephen A. Ross en 1977, la cual afirma que la combinación de fuentes de financiamiento permite minimizar el costo de capital, y maximizar el valor de mercado de la empresa (Tenjo, 2011), es decir, dependiendo de las fuentes de financiamiento a las que se recurra, será el comportamiento que tendrá el costo de capital y el valor de mercado en cada empresa.

El uso de deuda permite ahorrar impuesto, un uso elevado de esta, puede generarle a la empresa dificultades financieras (Mondragón, 2011), por lo tanto, cuando la empresa cuenta con un nivel de endeudamiento relativamente bajo, la probabilidad que se presenten dificultades financieras es baja y en esta situación, los beneficios de la deuda son superiores a sus costos, generando un aumento del valor de mercado de la empresa. Dentro del valor de mercado de la empresa, en una situación ideal de mercados, lo que determinaba dicho valor eran las inversiones, o bien, el tamaño y riesgo de los flujos de caja que generaban los activos de la empresa (Mondragón, 2011).

En caso contrario, es decir, cuando los costos de las dificultades financieras superan los beneficios fiscales de la deuda, se puede terminar destruyendo o disminuyendo su valor (Mayorga, 2011). Por lo tanto la estructura de capital óptima, corresponde al nivel de endeudamiento donde se igualan el valor del ahorro fiscal por deuda, con el valor de las dificultades financieras. 
Para la teoría del Trade-Off, el valor de la empresa no está dado por el rendimiento de los activos presentes de la misma, sino por el valor presente neto del rendimiento que podría obtener con futuras inversiones (Mondragón, 2011). En este sentido, la empresa busca una estructura óptima de capital, pues el nivel y las características de la deuda que la empresa contraiga limitarán las decisiones de inversión.

Este razonamiento permite explicar por qué las empresas no se endeudan tanto como les es posible. A la vez, las empresas no se financian exclusivamente con capital, pues al haber ciertos beneficios por mantener deuda en la estructura de capital como la deducción en el impuesto de renta de los intereses pagados no capitalizados, será necesario sopesar los beneficios posibles con los riesgos inherentes a esta fuente de recursos (Mondragón, 2011).

\section{METODOLOGÍA}

El estudio se realiza a artículos científicos, aplicados a empresas Latinoamericanas de diferentes sectores de mercado, publicados en un lapso de tiempo del año 2008 al 2016, los cuales aplicaron la Teoría del Trade-Off, como herramienta de análisis, para observar si responden a esta teoría en base a su comportamiento financiero y de financiamiento.

La metodología empleada fue documental, descriptiva, retrospectiva y con muestreo no probabilístico por conveniencia. Documental al realizar una búsqueda en artículos científicos publicados en las bases de datos Google Académico, Repositorio Institucional Pirhua, Educyt, Revistas EIA, Redalyc, Repository la Salle, Ecorfan, Scielo, dentro de un contexto latinoamericano, en un espacio temporal del 2008 al 2016. Descriptiva al obtener características de estas publicaciones en cuanto al año de publicación, autor, país, sector, metodología aplicada y resultados. Retrospectivo, al hacer el estudio durante un periodo definido de años atrás $(2008$ - 2016). Y no probabilístico por conveniencia, al seleccionar 20 artículos que abordaran el Trade-off, fueran latinoamericanos, que las empresas analizadas cotizaran en bolsa y su publicación fuera dentro del periodo de estudio.

\section{RESULTADOS}

A continuación, se presentan los resultados agrupados en tablas donde se muestra la información recabada.

Como se observa en la tabla 1 , el mayor sector analizado fue el terciario, seguido del secundario y finalmente el primario; sin embargo, existen 7 artículos que no especifican el sector abordado.

Tabla 1. Sectores estudiados por publicación

\begin{tabular}{|l|l|l|}
\hline \multicolumn{1}{|c|}{ SECTORES } & \multicolumn{1}{|c|}{ NÚMERO DE ARTICULOS } & \multicolumn{1}{|c|}{ SUBSECTORES } \\
\hline Primario (extracción) & 3 & $\begin{array}{l}\text { Agrícola } \\
\text { Agropecuario } \\
\text { Forestal }\end{array}$ \\
\hline
\end{tabular}




\begin{tabular}{|l|l|l|}
\hline Secundario (trasformación) & 4 & $\begin{array}{l}\text { Industrial } \\
\text { Automotor } \\
\text { Construcción } \\
\text { Inmobiliaria }\end{array}$ \\
\hline $\begin{array}{l}\text { Terciario (comercialización y y } \\
\text { servicio) }\end{array}$ & 6 & $\begin{array}{l}\text { Telecomunicaciones } \\
\text { Consumo } \\
\text { Comunicación } \\
\text { Salud }\end{array}$ \\
& & $\begin{array}{l}\text { Turismo } \\
\text { Cosméticos y Aseo }\end{array}$ \\
\hline No especifica & & $\begin{array}{l}\text { Empresas que cotizan en Bolsa de } \\
\text { Valores }\end{array}$ \\
\hline
\end{tabular}

Fuente: Elaboración propia.

Se observa en la tabla 2 que Colombia es el país en Latinoamérica donde se realiza mayor publicación artículos científicos relacionados con el análisis de la Teoría del Trade-Off. En cuanto a los autores, no hay coincidencia.

Tabla 2. Países que abordan estudio sobre trade off

\begin{tabular}{|l|l|c|}
\hline \multicolumn{1}{|c|}{ PAIS } & \multicolumn{1}{|c|}{ AUTORES } & FRECUENCIA \\
\hline Colombia & $\begin{array}{l}\text { Mejía (2015); Mogollón, López (2015); Orlando, Sattler, Bertoni, Domingo } \\
\text { (2015); Mondragón (2013); Vargas, Milena, Corredor (2013); Berlingeri } \\
\text { (2013); Mejía, Porras, Tirso (2012); Medina, Salinas, Ochoa, Molina (2012); }\end{array}$ & \\
& $\begin{array}{l}\text { Ortiz, Godoy (2012); Virgen, Rivera (2012); Rivera (2011); Mondragón } \\
\text { (2011); Wadnipar, Cruz (2008); }\end{array}$ & \\
\hline Perú & Garrido (2016). & 1 \\
\hline México & $\begin{array}{l}\text { Arredondo, Garza, Salazar (2015); Ortiz, Martínez, López, (2014); } \\
\text { Hernández, Ríos (2012). }\end{array}$ & 3 \\
\hline España & Cabrer, Rico (2015); Lajara, Mateos (2012). & 2 \\
\hline Chile & Gutiérrez, Aguayo, Panes (2014). & 1 \\
\hline Ecuador & Coronel, Vera (2015). & 1 \\
\hline
\end{tabular}

Fuente: Elaboración propia

En la tabla 3 se observa la clasificación de los subsectores por tamaño de empresa, destacando las medianas, seguidas de las pequeñas empresas.

Tabla 3. Tamaño de la empresa por subsector

\begin{tabular}{|l|l|l|}
\hline TAMAÑo DE LA EMPRESA & \multicolumn{1}{|c|}{$\begin{array}{c}\text { Número de } \\
\text { artículos }\end{array}$} & \multicolumn{1}{|c|}{ SUBSECTOR } \\
\hline Pequeña & 4 & $\begin{array}{l}\text { Agrícola } \\
\text { Agropecuario } \\
\text { Forestal } \\
\text { Consumo }\end{array}$ \\
\hline Mediana & 7 & $\begin{array}{l}\text { Industrial } \\
\text { Automotor } \\
\text { Construcción } \\
\text { Inmobiliaria } \\
\text { Cosméticos y Aseo } \\
\text { Salud }\end{array}$ \\
\hline & & \\
\hline
\end{tabular}




\begin{tabular}{|l|l|l|}
\hline & & Turismo \\
\hline Grande & 2 & $\begin{array}{l}\text { Telecomunicaciones } \\
\text { Comunicación }\end{array}$ \\
\hline No especifica & 7 & $\begin{array}{l}\text { Empresas que cotizan en } \\
\text { Bolsa de Valores }\end{array}$ \\
\hline
\end{tabular}

Fuente: Elaboración propia

En la tabla 4 se observa que el año 2015 es el de mayor publicación, seguido del 2012, denotando un patrón en el tiempo donde cada tres años la producción de artículos científicos sobre Trade-Off aumenta; como dato interesante, el límite inferior como el superior del periodo estudiado tienen solo una publicación dentro de esta muestra.

Tabla 4. Año de publicación

\begin{tabular}{|c|c|}
\hline AÑO & FRECUENCIA \\
\hline 2008 & 1 \\
\hline 2011 & 2 \\
\hline 2012 & 5 \\
\hline 2013 & 3 \\
\hline 2014 & 2 \\
\hline 2015 & 6 \\
\hline 2016 & 1 \\
\hline
\end{tabular}

Fuente: Elaboración propia

Se observa en la tabla 5 que los artículos analizados generan análisis basados principalmente en métodos estadísticos, excepto cuando se trata del sector primario, que lo hace a través de análisis financieros; los instrumentos de análisis detectados son dos estados financieros básicos (balance general y estado de resultados) en todos los sectores, excepto el primario, que los estudios no hacen referencia al instrumento empleado.

Tabla 5. Variables y métodos utilizados

\begin{tabular}{|c|c|c|c|}
\hline SECTOR & VARIABLES & METODOS & $\begin{array}{l}\text { INSTRUMENTO DE } \\
\text { ANALISIS }\end{array}$ \\
\hline Primario & $\begin{array}{l}\text { Tamaño } \\
\text { Oportunidades de crecimiento }\end{array}$ & $\begin{array}{l}\text { Ciclo de vida } \\
\text { Valor presente }\end{array}$ & No se especifica \\
\hline Secundario & $\begin{array}{l}\text { Tamaño } \\
\text { Apalancamiento } \\
\text { Rentabilidad }\end{array}$ & $\begin{array}{l}\text { Estadística inferencial } \\
\text { Método estadístico de Panel }\end{array}$ & $\begin{array}{l}\text { Balance general } \\
\text { Estado de Resultados }\end{array}$ \\
\hline Terciario & $\begin{array}{l}\text { Tamaño } \\
\text { Apalancamiento } \\
\text { Rentabilidad }\end{array}$ & $\begin{array}{l}\text { Estadística inferencial } \\
\text { Método estadístico de Panel }\end{array}$ & $\begin{array}{l}\text { Balance general } \\
\text { Estado de Resultados }\end{array}$ \\
\hline No especifica & $\begin{array}{l}\text { Tamaño } \\
\text { Apalancamiento } \\
\text { Rentabilidad } \\
\text { Oportunidades de crecimiento }\end{array}$ & Estadística inferencial & $\begin{array}{l}\text { Balance general } \\
\text { Estado de Resultados }\end{array}$ \\
\hline
\end{tabular}




\section{CONCLUSIONES}

De acuerdo a los resultados presentados, el sector mayoritariamente abordado en los artículos revisados es el terciario, es decir, las empresas de servicios; el que presenta una menor atención es el primario. Respecto a la región geográfica de estudio, se observó que el país que genera mayor número de estudios científicos es Colombia y el resto de los países que abordan estos estudios (México, España, Chile Ecuador y Perú), en un periodo de 10 años, es mínimo el aporte que generan a la investigación sobre la teoría del Trade-Off.

En el análisis por tamaño de las empresas destacan las medianas, en contra parte de las empresas grandes que son escasos los estudios que las abordan. En cuanto al análisis dentro del espacio temporal planteado (2008 - 2016) destaca el año 2015, seguido por el 2012, observando un patrón de aumento de producción cada tres años dentro del periodo revisado.

En cuanto a los métodos e instrumentos de análisis utilizados, destacan los análisis estadísticos basados en la información del balance general y estado de resultados, donde las variables mayormente usadas son tamaño, apalancamiento y rentabilidad.

\section{DISCUSIÓN}

De acuerdo con los resultados obtenidos del análisis realizado a los artículos científicos es estudio, se comenta lo siguiente:

1. En América Latina, el país que produce mayor número de análisis e investigación científica relativos a la teoría del Trade-off es Colombia, lo que hace inferir que para este país es relevante el análisis de sus empresas en cuanto a la estructura de capital. 2. Respecto al tamaño, es relevante profundizar en estudios posteriores por qué se enfocan estos estudios primordialmente a medianas. 3. Al observar principalmente análisis estadísticos inferenciales en los artículos, se asume que es la metodología más usada para el estudio de la estructura de capital. 4. Las variables de mayor frecuencia usadas en estudios latinoamericanos son tamaño, rentabilidad y apalancamiento.

\section{REFERENCIAS}

Arredondo, R., Catalina, E. y Salazar, J. (2015). Estructura óptima de capital: Análisis del TradeOff entre patrimonio y deuda para minimizar los costos de capital y maximizar el valor de la empresa, Vinculategica. 1(1), 1135-1149.

Berlingeri, H. (2014). ¿Trade-Off o Pecking Order? Una Investigación sobre las decisiones de financiamiento. Prenda, 4(8) 119-139. 
Cabrer, B. y Rico, P. (2015). Determinantes de la estructura financiera de las empresas españolas. Estudios de Economía Aplicada, 33(2), 513-531.

Garrido, M. (2016). Análisis de los factores que influyen en las decisiones de financiamiento empresarial cuando las empresas están sobre y subapalancadas. Tesis de pregrado en Economía, Universidad de Piura. Facultad de Ciencias Económicas y Empresariales Piura, Perú.

Gutiérrez, M., Aguayo, P. y Panes, P. (2014). Estructura de financiamiento de las empresas chilenas; aplicación del modelo de jerarquía. Horizontes Empresariales, 14(1), 7-19.

Hernández, G. y Ríos, H., (2012). Determinantes de la estructura financiera en la industria manufacturera: la industria de alimentos. Análisis Económico, 27(65), 101-121.

Lajara, N. y Mateos, A. (2012). Estructura financiera y logro empresarial en cooperativas agrarias: una aproximación empírica. Economía Agraria y Recursos Naturales. 12(2), 77101.

López, C. y Vera, P. (2015). Pecking Order y Trade-Off, aplicabilidad de teorías de reestructura de capital en el sector manufacturero bursátil del Ecuador.

Mayorga, J. (2011). La estructura financiera óptima en las PYMEs del sector industrial de la ciudad de Bogotá, Colombia. XVI Congreso Internacional de Contaduría Administración e Informática. México.

Medina, A., Salinas, J. Ochoa, L. y Molina, C. (2012). La estructura financiera de las empresas manufactureras colombianas, una mirada econométrica, macroeconómica y financiera. Perfil de Coyuntura Económica, 12(20), 155-176.

Mejía, A. (2015). La estructura de capital en las medianas empresas del departamento de Boyacá. Colombia, Apuntes del CENES, 34(59), 185-206.

Mejía, A., Porras, A. y Tirso, H. (2012). Análisis de la estructura financiera en las medianas empresas exportadoras de flores en un ambiente revaluacionistra. Desarrollo, Economía y Sociedad. 1(1), 15-26.

Mogollón, H. y López, C. (2015). Estructura de capital de las empresas del sector agropecuario en Colombia (2004-2013). Tesis. Universidad de la Salle. Facultad de Ciencias Económicas y Sociales. Colombia.

Mondragón, S. (2011). Marco conceptual de las teorías de la irrelevancia, trade off y de la jerarquía de las preferencias. Colombia.

Mondragón, S. (2011). Marco conceptual de las teorías de la irrelevancia, del trade-off y de la jerarquía de las preferencias. Cuaderno de Contabilidad, 12(30), 165-178 
Mondragón, S. (2013). Estructura de capital del sector automotor colombiano: una aplicación de la teoría de la jerarquía de las preferencias. Colombia, 14(34), 219-243.

Ortiz, R., Martínez, M. y López, I. (2014). Estructura de capital de las empresas del sector telecomunicaciones que cotizan en la BMV. Ecorfan. 4(-), 281-292.

Ortiz, V. y Rivera, J. (2012). Condiciones financieras que impactan la estructura financiera de la industria de cosméticos y aseo en Colombia, Cuadernos de Administración, 25(44), 175191.

Pérez, J., Sattler, S., Bertoni, M. y Domingo, D. (20115). Bases para un modelo de estructura de financiamiento en las pymes latinoamericanas, Cuaderno de Contabilidad, 16(40), 179204.

Rivera, J. (2011). Aporte de la Teoría de Agencia al puzzle de la estructura de capital de la empresa, Cuadernos de Administración 24(-), 129-167.

Tenjo, F. (2002). Determinantes de la estructura de capital en las empresas colombianas. Banco de la República Colombiana. Colombia.

Wadnipar, S. y Cruz, J. (2008). Determinación de la estructura de capital de las empresas colombianas. Soluciones de Postgrado EIA, 2008(1) 23-44.

Zambrano, S. y Acuña, G. (2013). Teoría del Pecking Order versus teoría del Trade-off para la empresa Coservicios S.A. E.S.P., Apuntes del CENES. 32(56), 205-236. 